\title{
Contrast mechanisms in polarization-sensitive Mueller-matrix optical coherence tomography and application in burn imaging
}

\author{
Shuliang Jiao, Wurong Yu, George Stoica, and Lihong V. Wang
}

\begin{abstract}
We investigate the various contrast mechanisms provided by polarization-sensitive (PS) Mueller-matrix optical coherence tomography (OCT). Our PS multichannel Mueller-matrix OCT is the first, to our knowledge, to offer simultaneously comprehensive polarization-contrast mechanisms, including the amplitude of birefringence, the orientation of birefringence, and the diattenuation in addition to the polarization-independent intensity contrast, all of which can be extracted from the measured Jones or the equivalent Mueller matrix. Theoretical analysis shows that when diattenuation is negligible, the roundtrip Jones matrix represents a linear retarder, which is the foundation of conventional PS-OCT, and can be calculated with a single incident polarization state, although the one-way Jones matrix generally represents an elliptical retarder; otherwise, two incident polarization states are needed. The experimental results obtained from rat skin samples, which conform well with the histology, show that Mueller OCT provides complementary structural and functional information on biological samples and reveal that polarization contrast is more sensitive to thermal degeneration of biological tissue than amplitude-based contrast. Thus, Mueller OCT has significant potential for application in the noninvasive assessment of burn depth. (C) 2003 Optical Society of America
\end{abstract}

OCIS codes: $120.2130 ; 170.4500 ; 260.5430 ; 170.1870$.

\section{Introduction}

Since it was first reported approximately a decade ago as a high-resolution noninvasive medical imaging modality, optical coherence tomography (OCT) has received tremendous attention. Several branches of OCT have been developed based on various contrast mechanisms, such as polarization ${ }^{1-6}$ and Doppler shift, ${ }^{7,8}$ in addition to the amplitude-based contrast in conventional OCT. OCT has found applications in imaging of the retina, cornea, gastrointestinal tract, artery, tooth, bladder, blood flow, and brain cortex. ${ }^{9}$ Another potential application of OCT is the evaluation of burns in biological tissue. ${ }^{10}$ The contrast of an OCT image is provided by the optical properties of a sample that modify the parameters of the light

The authors are with Texas A\&M University, 3120 TAMU, College Station, Texas 77843-3120. S. Jiao, W. Yu, and L. V. Wang (LWang@tamu.edu) are with the Optical Imaging Laboratory, Department of Biomedical Engineering. G. Stoica is with the Department of Pathobiology.

Received 18 January 2003; revised manuscript received 28 May 2003.

0003-6935/03/255191-07\$15.00/0

(C) 2003 Optical Society of America field, including the amplitude and the polarization state. The parameters characterizing the structurally isotropic or averaged optical properties ${ }^{11}$ of a sample include the absorption coefficient $\left(\mu_{\alpha}\right)$, scattering coefficient $\left(\mu_{s}\right)$, scattering anisotropy $(g)$, and refractive index $(n)$; and the parameters characterizing the polarization properties of a sample include birefringence (amplitude $\delta n$, orientation, and ellipticity) and diattenuation (amplitude $D$, orientation, and ellipticity), which provide polarization-based contrast in polarization-sensitive OCT (PS-OCT).

Because of the interference-based heterodyne detection scheme used in OCT, a scattering sample acts as a nondepolarizing medium. ${ }^{4}$ The polarization properties of a nondepolarizing sample can be completely characterized by either a Mueller matrix or a Jones matrix, and the two matrices are equivalent. ${ }^{12}$ Therefore, to provide comprehensive information about polarization of a sample, the most general PSOCT should measure the Jones or the Mueller matrix. Upon acquisition of the Jones or the Mueller matrix, any polarization parameters can be extracted. We define Mueller-matrix OCT as PS-OCT that can measure the Mueller or the Jones matrix of a sample. Therefore, Mueller-matrix OCT is the most general form of PS-OCT. 
In this paper, we investigate the various contrast mechanisms provided by Mueller-matrix OCT. The properties of the round-trip Jones matrix are analyzed for conditions with and without diattenuation in a sample. The analyses indicate that when diattenuation is negligible, one incident polarization state is adequate for the acquisition of the Jones matrix. When diattenuation cannot be neglected, two incident polarization states are necessary, and the transpose symmetric property of the round-trip Jones matrix (first discovered by our group ${ }^{5}$ ) offers a critical condition for the calculation of the Jones matrix correctly. Experimental results with biological samples are presented.

\section{Polarization-Based Contrast}

Diattenuation is a description of the dependence of transmittance on the incident polarization states and is defined as

$$
D=\left(P_{q}^{2}-P_{r}^{2}\right) /\left(P_{q}^{2}+P_{r}^{2}\right),
$$

where $P_{q}$ and $P_{r}$ represent the amplitude transmittances for the two orthogonal eigenpolarizations of a polarization element. Therefore, diattenuation provides anisotropic amplitude-based contrast, as it incurs no phase retardation. Birefringence is a description of the anisotropic dependence of the phase velocity of light in a sample on the incident polarization states. The phase retardation of a light field, induced by the local birefringence between the two orthogonal eigenpolarizations, can be expressed as

$$
d \phi=\bar{k} \delta n\left(L_{s}{ }^{\prime}\right) d L_{s}{ }^{\prime},
$$

where $\bar{k}$ is the wave vector corresponding to the central wavelength of the incident light in vacuum; $L_{s}{ }^{\prime}$ is the physical path length that the light travels in the birefringent medium; $\delta n\left(L_{s}{ }^{\prime}\right)$ is the local birefringence; and $d L_{s}{ }^{\prime}$ is the local physical path length. The phase retardation provides a unique phase-based polarization-contrast mechanism reflecting the amplitude of birefringence, which exists in various biological components such as collagen, keratin, myelin, and elastic fibers. Because highly birefringent collagen is a predominant structural component in most biological tissues, this intrinsic contrast mechanism is prevalent in the biomedical applications of Mueller OCT. In addition, many degenerative processes of biological tissues alter birefringence and should, thus, be detectable by Mueller-matrix OCT.

In a PS-OCT system, the detected variation of the polarization state of the scattered light in reference to the incident light is affected by the round-trip polarization effect of a sample, which can be characterized with a round-trip Jones matrix $\left(\mathbf{J}_{2}\right)$. We will use subscripts 1 and 2 to describe the one-way and round-trip parameters, respectively. After acquisition of the round-trip Jones matrix, the round-trip retardation $\left(\phi_{2}\right)$ and diattenuation $\left(D_{2}\right)$ for each pixel of the OCT image can be calculated with the following formulas, ${ }^{13}$

$$
\begin{aligned}
\phi_{2} & =2 \cos ^{-1}\left\{\frac{1}{2} \frac{\left|\operatorname{tr} \mathbf{J}_{2}+\left[\operatorname{det} \mathbf{J}_{2} /\left|\operatorname{det} \mathbf{J}_{2}\right|\right] \operatorname{tr} \mathbf{J}_{2} *\right|}{\left[\operatorname{tr}\left(\mathbf{J}_{2} * \mathbf{J}_{2}\right)+2\left|\operatorname{det} \mathbf{J}_{2}\right|\right]^{1 / 2}}\right\}, \\
D_{2} & =\left\{1-\frac{4\left|\operatorname{det} \mathbf{J}_{2}\right|^{2}}{\left[\operatorname{tr}\left(\mathbf{J}_{2} * \mathbf{J}_{2}\right)\right]^{2}}\right\}^{1 / 2},
\end{aligned}
$$

respectively, where $*$, tr, and det represent the Hermitian (transpose conjugate), trace, and determinant of the matrix, respectively. The fast eigenvector of $\mathbf{J}_{2}$ at each pixel of the OCT image,

$$
E_{2}=\left[\begin{array}{c}
E_{2 h} \\
E_{2 v}
\end{array}\right],
$$

can be calculated through standard algorithms. The orientation of the fast axis can thus be calculated as

$$
\theta_{2}=\arctan \left(\frac{E_{2 v}}{E_{2 h}}\right) .
$$

\section{Calculation of the Round-Trip Jones Matrix}

The round-trip Jones matrix $\mathbf{J}_{2}$ can be expressed with the one-way Jones matrix $\left(\mathbf{J}_{1}\right)$, according to the Jones reversibility theorem, as

$$
\mathbf{J}_{2}=\mathbf{J}_{1}^{T} \mathbf{J}_{1},
$$

where the superscript $T$ represents the transpose operation. A polarization element is called homogeneous when the two eigenvectors of its Jones matrix are orthogonal. A retarder is called elliptical when its eigenpolarizations are elliptical polarization states. A linear retarder is a special case in which the eigenpolarizations are linear, and a Faraday rotator is another special case in which the eigenpolarizations are circular. We can prove that when two or more linear retarders are cascaded, the overall retarder is generally elliptical unless the axes of the retarders are aligned. Except in some special samples, the orientations of the birefringent fibers in biological samples, take skin, for example, are not collinear, and as a result, $\mathbf{J}_{1}$ generally represents a homogeneous elliptical retarder if diattenuation is negligible in the sample.

When diattenuation is negligible in a sample, $\mathbf{J}_{1}$ can be expressed as

$$
\begin{aligned}
\mathbf{J}_{1}\left(\phi_{1}, \theta_{1}, \delta_{1}\right) & =\left[\begin{array}{ll}
\cos \left(\phi_{1} / 2\right)+i \sin \left(\phi_{1} / 2\right) \cos 2 \theta_{1} & i \sin \left(\phi_{1} / 2\right) \sin 2 \theta_{1} \exp \left(-i \delta_{1}\right) \\
i \sin \left(\phi_{1} / 2\right) \sin 2 \theta_{1} \exp \left(i \delta_{1}\right) & \cos \left(\phi_{1} / 2\right)-i \sin \left(\phi_{1} / 2\right) \cos 2 \theta_{1}
\end{array}\right] \\
& =\left[\begin{array}{cc}
J_{1}(1,1) & J_{1}(1,2) \\
-J_{1}(1,2)^{*} & J_{1}(1,1)^{*}
\end{array}\right] .
\end{aligned}
$$


The fast and slow eigenvectors are

$$
\left[\begin{array}{c}
\cos \theta_{1} \\
\sin \theta_{1} \exp \left(i \delta_{1}\right)
\end{array}\right]
$$

and

$$
\left[\begin{array}{c}
-\sin \theta_{1} \exp \left(-i \delta_{1}\right) \\
\cos \theta_{1}
\end{array}\right]
$$

respectively, where $\theta_{1}$ is an auxiliary angle and $\delta_{1}$ represents the phase difference between the two components of the fast eigenvector. $\phi_{1}$ is the phase difference between the two eigenvalues (the retardation). The azimuth $\left(\alpha_{1}\right)$ of the major axis of its fast eigenpolarization can be expressed as $\tan \left(2 \alpha_{1}\right)=\tan \left(2 \theta_{1}\right) \cos \delta_{1}$. If $\delta_{1}=0, \mathbf{J}_{1}$ is transpose symmetric, representing a linear retarder, and $\theta_{1}$ represents the orientation of the fast axis.

From Eq. (5), we have $\mathbf{J}_{2}=\mathbf{J}_{2}{ }^{T}$, i.e., $\mathbf{J}_{2}$ is transpose symmetric. As a result, $\mathbf{J}_{2}$ represents a linear retarder, and we can thus conclude that the round-trip transformation effect of an elliptical retarder is equivalent to the one-way transformation of a linear retarder. This conclusion is the foundation of conventional PS-OCT, in which a sample is treated as a linear retarder. Since only two parameters are needed to characterize a linear retarder, the number of parameters needed to characterize the round-trip polarization properties of a sample is reduced to two. This conclusion allows the acquisition of this type of round-trip Jones matrix with only one incident polarization state. For an incident polarization state

$$
\mathbf{E}_{i}=\left[\begin{array}{c}
E_{i h} \\
E_{i v}
\end{array}\right],
$$

the output polarization state

$$
\mathbf{E}_{o}=\left[\begin{array}{c}
E_{o h} \\
E_{o v}
\end{array}\right]
$$

detected by PS-OCT can be expressed as

$$
\left[\begin{array}{c}
E_{o h} \\
E_{o v}
\end{array}\right]=\mathbf{J}_{2}\left[\begin{array}{c}
E_{i h} \\
E_{i v}
\end{array}\right] .
$$

Because of the orthonormal transformation property of $\mathbf{J}_{2}$, the inherent property of a retarder, we also have

$$
\left[\begin{array}{c}
E_{o v}^{*} \\
-E_{o h}^{*}
\end{array}\right]=\mathbf{J}_{2}\left[\begin{array}{c}
E_{i v}^{*} \\
-E_{i h}^{*}
\end{array}\right] .
$$

The round-trip Jones matrix can thus be calculated as

$$
\mathbf{J}_{2}=\left[\begin{array}{cc}
E_{o h} & E_{o v}^{*} \\
E_{o v} & -E_{o h} *
\end{array}\right]\left[\begin{array}{cc}
E_{i h} & E_{i v}^{*} \\
E_{i v} & -E_{i h}^{*}
\end{array}\right]^{-1} .
$$

When diattenuation cannot be neglected in a sample, one incident polarization state is not sufficient to acquire its round-trip Jones matrix because five real parameters $\left[\phi_{2}, \theta_{2}\right.$, amplitude transmittances $\left(P_{q 2}\right.$ and $\left.P_{r 2}\right)$, and the orientation of diattenuation $\left(\theta_{d 2}\right)$ ] are needed to characterize such a system. Therefore, at least two incident polarization states, either applied at the same time or applied sequentially, are required. The transpose symmetry in the roundtrip Jones matrix is critical for eliminating the arbitrary phase difference between the two measured Jones vectors corresponding to the two incident polarization states to yield the correct Jones matrix. This arbitrary phase difference can be caused either by the nonidentity of the power spectra when two light sources are used or by the imperfection of the longitudinal scanning mechanism when the two incident polarization states are applied sequentially. Because it ignores the diattenuation effect completely, conventional PS-OCT is not valid for biological samples possessing diattenuation and cannot provide diattenuation contrast.

\section{Experiment}

We have built a novel multichannel Mueller OCT, 5,6 which can acquire the Jones matrix of a sample with a single scan for each one-dimensional depth image (A line image). The Jones matrix can be further transformed into an equivalent Mueller matrix. The Mueller matrix is preferred because its first element, $M_{00}$, represents the intensity transformation property of a sample and is free of both the effects of the sample polarization and the polarization state of the incident light. Therefore, a Mueller matrix reveals the real morphologic structure as well as the polarization-based features of a sample.

The tail of a rat was imaged in situ with Mueller OCT after the skin was shaved and scrubbed with glycerin. The OCT and polarization-histologic images are shown in Figs. 1(a)-1(f). There are no significant differences between the $M_{00}$ image [Fig. 1(b)] and the conventional OCT image for this particular sample [Fig. 1(a)], both of which are amplitude based. The effect of polarization on a conventional OCT image depends on several parameters, for example, the incident polarization state, the value and orientation of the birefringence, and the accumulated phase retardation. When fringes are present in the conventional OCT image, the difference between these two images is dramatic. ${ }^{5}$ The intensity and retardation images reveal different characteristics of the sample. The intensity images clearly reveal the boundaries of the structures in the epidermis and only the shallow dermal region. In contrast, the retardation image [Fig. 1(c)] reveals the distribution of birefringent components deeper into the dermis. The absolute value of the retardation difference between each pixel and its previous pixel in the same A line is calculated to obtain a differential retardation image [Fig. 1(d)]. The birefringent regions (corresponding to the superficial keratin layer and collagen-rich dermal papillae) and nonbirefringent regions (corresponding to fat and the living epidermis) are shown more clearly in the differential retardation image than in the raw retardation image. The im- 

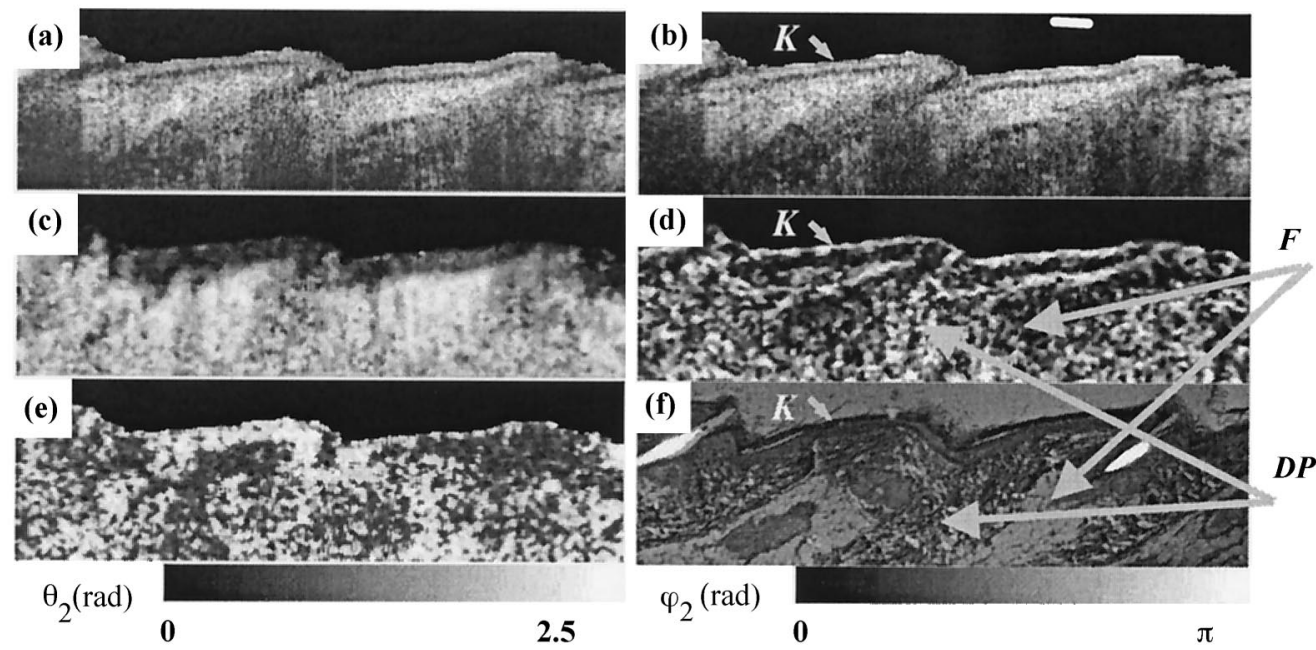

Fig. 1. (a) Conventional OCT image (in logarithmic scale), (b) intensity image $\left(M_{00}\right.$, in logarithmic scale), (c) retardation image, (d) differential retardation image, (e) image of the orientation of the fast axis, (f) polarization histologic image of an in situ rat tail. The height of each image is $750 \mu \mathrm{m}$. The gray scales are for the orientation $\left(\theta_{2}\right)$ and the retardation $\left(\phi_{2}\right)$ images. The conventional OCT image was obtained with vertical linear polarization states for both the incident and the reference beams. $F$, fat; $K$, keratin; $D P$, dermal papilla.

age of the orientation of the fast axis [Fig. 1(e)] revealed structures that we believe to be related to the distribution of the orientation of the birefringent fibers (collagen and keratin). In the figure, we can see that the orientation of the fast axis varies from region to region as also observed in the polarization histology. Although the amplitudeand phase-based polarization signals should have comparable signal-to-noise ratios because they are computed from the same measurements, the contrast-to-noise ratio can be different depending on the availability of the two contrasts in the sample; therefore, the two contrast mechanisms can provide information into different depths.

To evaluate the sensitivity of the phase-based polarization contrast in burn-depth determination, we imaged an ex vivo skin sample-from a rat bellycontaining a burn lesion. The burn lesion was made by touching the skin with a heated (approximately
$100{ }^{\circ} \mathrm{C}$ ) electric iron for less than $1 \mathrm{~s}$. The calculated intensity image, the retardation image, the diattenuation image and the histological image are shown in Figs. 2(a)-2(d). The burn region cannot be identified in the intensity image; but it can be clearly seen with marked contrast in the retardation and diattenuation images as verified by the polarization histological image.

Figure 3 shows the depth profiles of retardation of the burn and normal regions, respectively. Each curve is an average of 10 profiles in the central area of the burn region and in the normal region to the right side of the burn region. The loss of birefringence in the burn region compared with the normal tissue can be seen clearly. This figure further demonstrates that phase-based polarization contrast provides a sensitive mechanism for evaluating thermal degeneration of biological tissue. Because birefringence and diattenuation are related to the function of
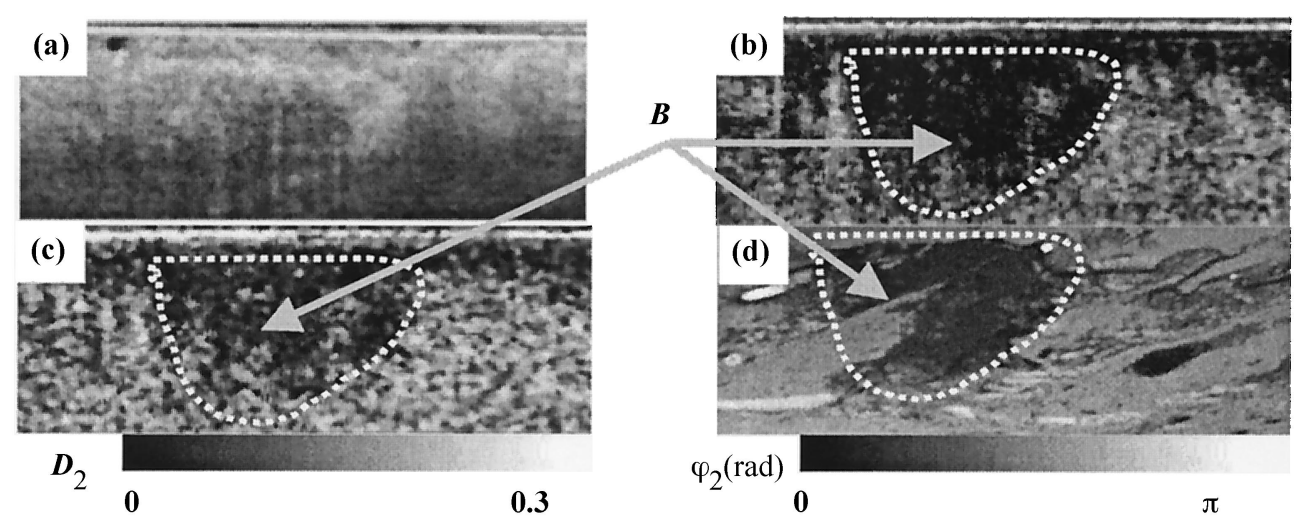

Fig. 2. (a) Intensity image $\left(M_{00}\right.$, in logarithmic scale), (b) retardation image, (c) diattenuation image, (d) polarization histologic image of a piece of ex vivo rat skin with a burn lesion. The height of each image is $750 \mu \mathrm{m}$. The gray scales are for the retardation $\left(\phi_{2}\right)$ and the diattenuation $\left(D_{2}\right)$ images. $B$, burn region. 


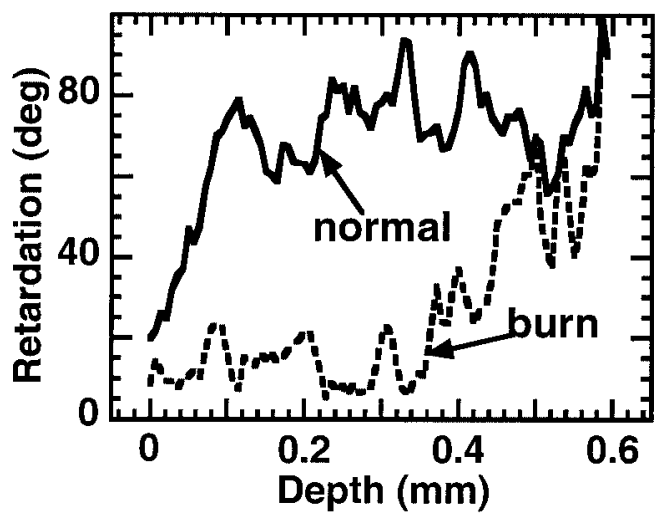

Fig. 3. Average of 10 depth profiles of the retardation around the center of the burn area and the normal region to the right of the burn area.

several kinds of biological component such as collagen, Mueller OCT is a type of functional imaging.

\section{Discussion}

The differences between conventional OCT and Mueller OCT in their sensitivities to different optical properties of a sample result from their different contrast mechanisms. Conventional OCT is an amplitude-based detection system, which detects the local relative variations of path-length-resolved reflectance from tissues. By modifying an existing theoretical model of $\mathrm{OCT}^{14}$ to include the effect of polarization, we can express the signal in conventional OCT as

$$
\begin{aligned}
\tilde{I}_{d}\left(L_{r}\right)= & 2\left(I_{s} I_{r}\right)^{1 / 2} \int_{-\infty}^{\infty}\left[R\left(L_{s}\right)\right]^{1 / 2} \cos \left[\beta\left(L_{s}\right)\right] \exp \\
& \times\left[-4\left(\Delta L / L_{c}\right)^{2}\right] \cos (\bar{k} \Delta L) d L_{s},
\end{aligned}
$$

where $L_{\mathrm{s}}$ and $L_{\mathrm{r}}$ are the round-trip optical path lengths of the sample and reference arms, respectively; $\Delta L=L_{s}-L_{r}$ is the round-trip optical pathlength difference; $L_{\mathrm{c}}$ is the coherence length of the light source; $I_{\mathrm{r}}$ is the intensity of the reference beam; $I_{\mathrm{S}}$ is the reflected intensity of the sample $\operatorname{arm} ; R\left(L_{\mathrm{s}}\right)=$ $\left[\mathrm{d} I_{\mathrm{s}}\left(L_{\mathrm{s}}\right) / \mathrm{d} L_{\mathrm{s}}\right] / I_{\mathrm{s}}$ is the path-length-resolved reflectance of the sample; and $\beta\left(L_{s}\right)$ is an equivalent angle between the polarization states of the reference and the backscattered sample beams, defined as

$$
\cos \left[\beta\left(L_{s}\right)\right]=\left\langle\mathbf{E}_{s}\left(L_{s}\right) \cdot \mathbf{E}_{r}\right\rangle /\left(\left|\mathbf{E}_{s}\left(L_{s}\right) \| \mathbf{E}_{r}\right|\right),
$$

where $\mathbf{E}_{s}\left(L_{s}\right)$ and $\mathbf{E}_{r}$ are the electric vectors of the sample and the reference beams, respectively, and the angle brackets denote a time average. The integrand is nonzero mainly in the interval $|\Delta L| \leq L_{c}$. The integration produces a significant value only when $R\left(L_{s}\right)$ varies sharply across a dimension of $L_{c}$; otherwise, the integral tends to be zero because of the cosine term in the integrand. A sharp variation of $R\left(L_{s}\right)$ is caused by interfaces between regions of different optical properties. Conventional OCT is, in principle, very sensitive to discontinuity of the refrac- tive index $(\Delta n)$ as a result of specular reflection. As studied by Pan et al., ${ }^{14,15}$ conventional OCT is also sensitive to variations of the anisotropy $(\Delta g)$ and the scattering coefficient $\left(\Delta \mu_{\mathrm{s}}\right)$, but it is insensitive to variation of the absorption coefficient $\left(\Delta \mu_{\mathrm{a}}\right)$. We can see in Eq. (10) that the polarization effect of a sample contributes to the recorded conventional OCT signal as an amplitude modulation and is superimposed on the backreflection effect; consequently, conventional OCT has difficulty in separating the polarization effect from the real morphologic effect of the sample.

To account for the meanings of the measured retardation image, we can divide each depth scan into a number of homogenous segments, each of which has a length less than the axial resolution; each segment can be characterized by a Jones matrix $\mathbf{J}_{1}(i)(i=1,2$, ...), which is a function of the equivalent local birefringence $[\delta n(i)]$, orientation of the fast axis $\left[\theta_{1}(i)\right]$, amplitude transmittances $\left[P_{q 1}(i)\right.$ and $\left.P_{r 1}(i)\right]$, and orientation of the diattenuation $\left[\theta_{d 1}(i)\right]$. For single backscattering and even multiple small-angle scattering, the equivalent round-trip Jones matrix of contiguous $m$ segments of the sample from the surface to the $m$ th segment can be expressed as

$$
\mathbf{J}_{2}^{(m)}=\prod_{i=1}^{m} \mathbf{J}_{1}^{\mathrm{T}}(\mathrm{i}) \prod_{i=m}^{1} \mathbf{J}_{1}(i) .
$$

The equivalent round-trip parameters for the $m$ segments, such as the retardation $\phi_{2}^{(m)}$, orientation of the fast axis $\theta_{2}^{(m)}$, and diattenuation, can be calculated from $\mathbf{J}_{2}^{(m)}$. When $\theta_{1}(1)=\theta_{d 1}(1)=\theta_{1}(2)=\theta_{d 1}(2)=$ $\ldots=\theta_{1}(m)=\theta_{d 1}(m)$, if $\phi_{2}^{(m)} \leq \pi, \phi_{2}^{(m)}$ in the retardation image increases with depth, whereas $\theta_{2}^{(m)}$ keeps constant; if $\phi_{2}^{(m)}$ covers a range greater than $\pi$, it causes fringes in both the retardation and the orientation images because a retarder $\mathbf{J}\left(\phi_{2}^{(m)}+\pi, \theta_{2}^{(m)}\right)$ is equivalent to a retarder $\mathbf{J}\left(\pi-\phi_{2}^{(m)}, \theta_{2}^{(m)} \pm \pi / 2\right)$, $\left(\phi_{2}^{(m)}, \theta_{2}^{(m)} \in[0, \pi]\right)$, a phenomenon observed in the retardation and orientation images of samples like porcine tendon. ${ }^{5}$ In this case, the differential retardation image reflects a map of the local birefringence. Otherwise, $\phi_{2}^{(m)}$ and $\theta_{2}^{(m)}$ are also functions of both $\theta_{1}(i)$ and $\theta_{d 1}(i)$ in the optical path, making the retardation image complex to interpret rigorously unless the local polarization properties can be calculated, which is possible only with Mueller OCT.

The Jones matrix of the first pixel of each A line represents the round-trip Jones matrix of the first segment, i.e., $\mathbf{J}_{2}^{(1)}=\mathbf{J}_{1}^{T}(1) \mathbf{J}_{1}(1)$. If $\mathbf{J}_{1}(1)$ can be calculated from $\mathbf{J}_{2}(1)$ by developing some effective algorithms, the first segment can be peeled off to yield the round-trip Jones matrix of the second segment, $\mathbf{J}_{1}{ }^{T}$ (2) $\mathbf{J}_{1}(2)=\left[\mathbf{J}_{1}^{T}(1)\right]^{-1} \mathbf{J}_{2}^{(2)} \mathbf{J}_{1}^{-1}$ (1). By use of this strategy layer by layer, the one-way Jones matrix of each segment can thus be extracted, and the images of the local polarization parameters can be calculated, which should be free of fringes because the retardation of each segment should be much less than $\pi$. This algorithm is important in fiber-based PSOCT system for eliminating the polarization dis- 


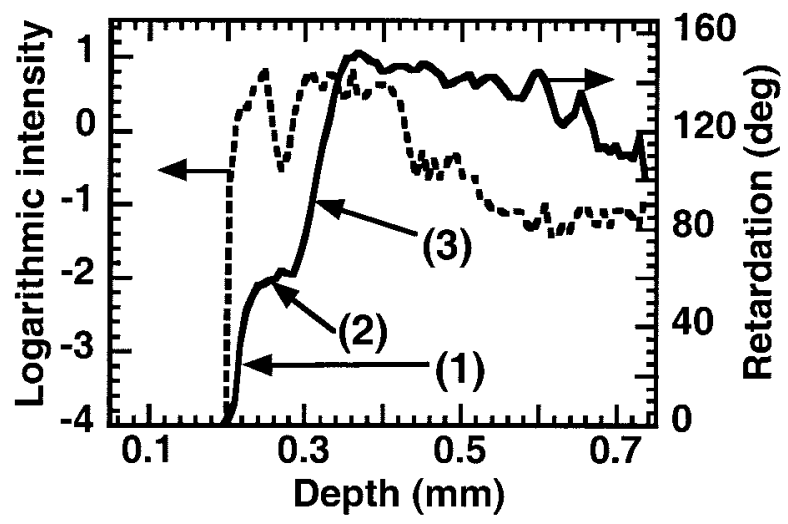

Fig. 4. Averaged depth profiles of the intensity (in logarithmic scale) and retardation over the region marked with a horizontal white bar in Fig. 1(b). Labels (1), (2), and (3), layers revealed.

tortions on the measured polarization-based images caused by the sampling fiber. ${ }^{16}$

Unlike amplitude-based contrast, phase-based polarization contrast is insensitive to a boundary caused by $\Delta n$, and, as a result, a boundary in the intensity image may not be reflected in the retardation image unless there is a corresponding difference of $\delta n$ across the boundary, the value of which also determines the sharpness of a boundary in the retardation image. Because of the integration over depth, a boundary caused by $\delta n$ in the retardation image may not be as sharp as the corresponding boundary caused by $\Delta n$ in the intensity image. However, as can be seen in Fig. 2(b), the interface between two regions of different $\delta n$ can be distinguished with sufficient accumulated contrast within a few coherence lengths. Figure 4 shows the depth profiles of intensity and retardation averaged laterally over the range marked by the white bar in Fig. 1(b). The retardation curve revealed several layers from the surface down into the skin. Layers (1) and (3) are highly birefringent, indicating the keratin in the epidermis and the dermal papilla, respectively. Layer (2) has almost no birefringence, which likely corresponds to the living epidermis.

We regard the apparent phase retardation induced by mechanisms other than birefringence as the background of the phase-based polarization contrast. Besides birefringence, scattering can also alter the polarization state of light and cause phase retardation. Alteration of the polarization state of the propagating light depends on the geometry and the refractive index of the scattering particles. ${ }^{17,18}$

\section{Conclusion}

In summary, a unique feature of Mueller OCT is its capability of separating various contrast mechanisms, in which the amplitude-based contrast is sensitive to the boundaries formed primarily by regions of different indexes of refraction, whereas the phasebased polarization contrast and the orientation-based contrast originate from the components of biological tissues with optical polarization effect. Experimen- tal results show that phase-based polarization contrast is more sensitive to thermal degeneration of biological tissues than amplitude-based contrast. The combination of amplitude-based contrast with phase-based polarization contrast and the orientation-based contrast provides more comprehensive information about biological tissues. Phase-based polarization contrast is a promising imaging mechanism for assessing burn depth in vivo.

We thank $\mathrm{G}$. $\mathrm{Ku}$ for help in preparing samples. This project was sponsored in part by the National Institutes of Health, grants R21 RR15368 and R01 EB000712; by the National Science Foundation, grant BES-9734491; and by the Texas Higher education Coordinating Board, grant 000512-0063-2001.

\section{References}

1. M. R. Hee, D. Huang, E. A. Swanson, and J. G. Fujimoto, "Polarization-sensitive low-coherence reflectometer for birefringence characterization and ranging," J. Opt. Soc. Am. B 9, 903-908 (1992).

2. J. F. de Boer, T. E. Milner, M. J. C. van Gemert, and J. S. Nelson, "Two-dimensional birefringence imaging in biological tissue by polarization-sensitive optical coherence tomography," Opt. Lett. 22, 934-936 (1997).

3. G. Yao and L.-H. V. Wang, "Two-dimensional depth-resolved Mueller matrix characterization of biological tissue by optical coherence tomography," Opt. Lett. 24, 537-539 (1999).

4. S. Jiao, G. Yao and L.-H. V. Wang, "Depth-resolved twodimensional Stokes vectors of backscattered light and Mueller matrices of biological tissue measured with optical coherence tomography," Appl. Opt. 39, 6318-6324 (2000).

5. S. Jiao and L.-H. V. Wang, "Two-dimensional depth-resolved Mueller matrix of biological tissue measured with double-beam polarization-sensitive optical coherence tomography," Opt. Lett. 27, 101-103 (2002).

6. S. Jiao and L.-H. V. Wang, "Jones-matrix imaging of biological tissues with quadruple-channel optical coherence tomography," J. Biomed. Opt. 7, 350-358 (2002).

7. Z. Chen, T. E. Milner, D. Dave, and J. S. Nelson, "Optical Doppler tomography imaging of fluid flow velocity in highly scattering media," Opt. Lett. 22, 64-66 (1997).

8. J. A. Izatt, M. D. Kulkarni, S. Yazdanfar, J. K. Barton, and A. J. Welsh, "In vivo bidirectional color Doppler flow imaging of picoliter blood volumes using optical coherence tomography," Opt. Lett. 22, 1439-1441 (1997).

9. S. A. Boppart, M. E. Brezinski, C. Pitris, and J. G. Fujimoto, "Optical coherence tomography for neurosurgical imaging of human intracortical melanoma," Neurosurgery 43, 1992-1998 (1998).

10. B. H. Park, C. Saxer, S. M. Srinivas, J. S. Nelson, and J. F. de Boer, "In vivo burn depth determination by high-speed fiberbased polarization sensitive optical coherence tomography," J. Biomed. Opt. 6, 474-479 (2001).

11. G. Marquez, L.-H. Wang, S.-P. Lin, J. A. Schwartz, and S. L. Thomsen, "Anisotropy in the absorption and scattering spectra of chicken breast tissue," Appl. Opt. 37, 798-804 (1998).

12. C. Brosseau, Fundamentals of Polarized Light: A Statistical Optics Approach (Wiley, New York, 1998).

13. S. Y. Lu and R. A. Chipman, "Homogenous and inhomogenous Jones matrix,” J. Opt. Soc. Am. A 11, 766-772 (1994).

14. Y. Pan, R. Birngruber, J. Rosperich, and R. Engelhardt, "Lowcoherence optical tomography in turbid tissue: theoretical analysis," Appl. Opt. 34, 6564-6574 (1995). 
15. Y. Pan, R. Birngruber, and R. Engelhardt, "Contrast limits of coherence-gated imaging in scattering media," Appl. Opt. 36, 2979-2983 (1997).

16. S. Jiao, W. Yu, G. Stoica, and L.-H. V. Wang, "Optical-fiberbased Mueller optical coherence tomography," Opt. Lett. 28, 1206-1208 (2003).
17. J. M. Schmitt and S. H. Xiang, "Cross-polarized backscatter in optical coherence tomography of biological tissue," Opt. Lett. 23, 1060-1062 (1998).

18. M. I. Mishchenko and J. W. Hovenier, "Depolarization of light scattered by randomly oriented nonspherical particles," Opt. Lett. 20, 1356-1358 (1995). 